\title{
A Double Whammy of Mycotic Aneurysms and Acquired Dysfibrinogenemia in a Patient with Septicemia
}

\author{
${ }^{*}$ Chun Yang $\operatorname{Sim}^{1}$ (D), ${ }^{*}$ Ching Soong $\mathrm{Khoo}^{2}$ (D), Ruslinda Mustafar ${ }^{2}$ (D), Jia Ning Chai ${ }^{3}$ (1)
}

\begin{abstract}
${ }^{1}$ Faculty of Medicine and Health Sciences, Universiti Sarawak Malaysia, Sarawak, Malaysia ${ }^{2}$ Department of Medicine, Universiti Kebangsaan Malaysia Medical Centre, Kuala Lumpur, Malaysia. ${ }^{3}$ Department of Radiology, Universiti Kebangsaan Malaysia Medical Centre, Kuala Lumpur, Malaysia *Both authors contributed equally to this work.
\end{abstract}

A 65-year-old woman with immunoglobulin A nephropathy presented with a 2-day history of fever and right lower limb swelling. She had been treated with cyclophosphamide for a recent relapse 5 days before the current presentation. Apart from a body temperature of $38^{\circ} \mathrm{C}$, her other vital signs were fairly stable. Her right lower limb, particularly the thigh, was swollen, erythematous, and mildly tender. Review of other systems was unremarkable. Blood tests revealed elevated levels of septic markers and presence of group A beta-hemolytic streptococci in the blood culture. She was treated with intravenous ampicillin/sulbactam for bacteremia. On the 12th day of hospitalization, her right thigh became more swollen and painful. The computed tomography showed a large heterogeneous retroperitoneal hematoma measuring $4.1 \mathrm{~cm} \times 14.2 \mathrm{~cm} \times 17.2 \mathrm{~cm}$ and another hematoma within the right gluteus medius muscle measuring $8.5 \mathrm{~cm} \times 14.6 \mathrm{~cm} \times 30 \mathrm{~cm}$ (Figure 1). The conventional angiogram showed an aneurysm from the right inferior pancreaticoduodenal artery with active bleeding, which was successfully secured

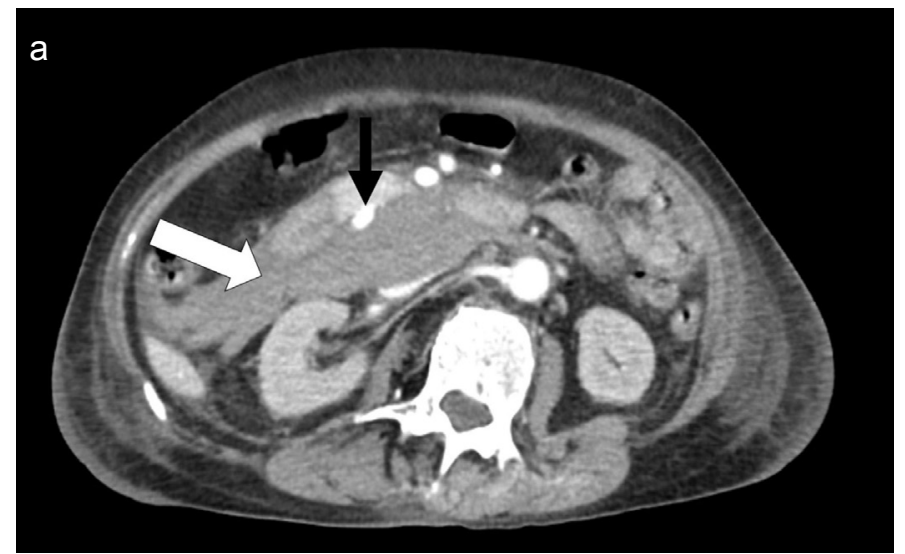

by arterial embolization (Figure 2. a, b). The right gluteal region was not intervened in the hope of achieving hemostasis through the tamponade effect of the hematoma. The embolization procedure was complicated with persistent oozing from the femoral puncture site. Repeated activated partial thromboplastin time (aPTT) was prolonged (>180 seconds), which was not corrected by the mixing test. Coagulopathy workup showed markedly prolonged thrombin time (TT) of 216.8 seconds, fibrinogen level of $2.14 \mathrm{~g} / \mathrm{L}$ (normal range, 1.36-4.65 g/L), and elevated levels of factors VIII and IX of $384 \%$ and $185 \%$, respectively. Acquired dysfibrinogenemia was highly suspected. The fibrinogen activity-to-antigen ratio was not calculated because the patient did not agree to perform the test. She was then treated with multiple transfusions of fresh frozen plasma, cryoprecipitate, packed cells, and desmopressin to achieve hemostasis. On the 16th day, she was found to have active bleeding from an aneurysm arising from a branch of the right inferior gluteal artery, which was then successfully treated with arterial embolization

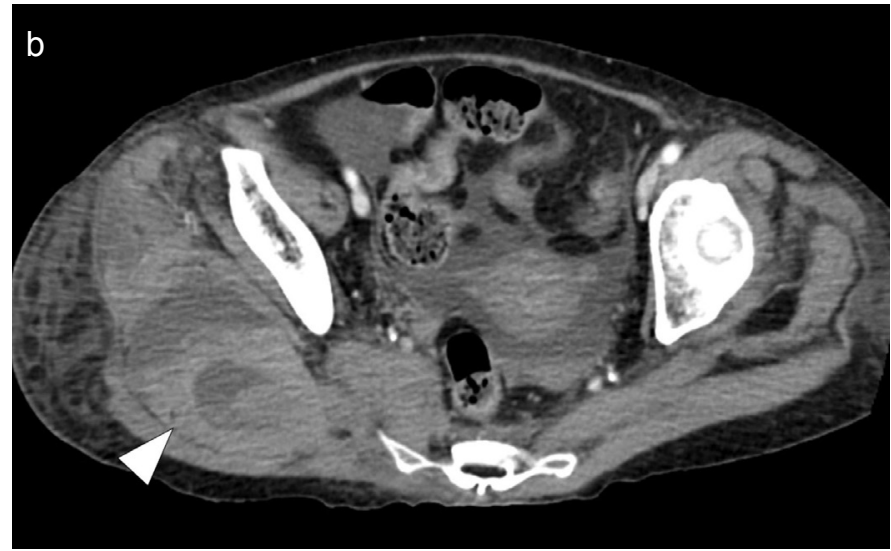

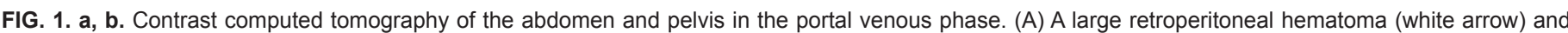

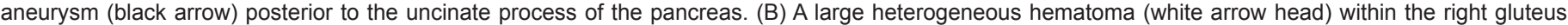
medius muscle

Address for Correspondence: Ching Soong Khoo, Department of Medicine, Universiti Kebangsaan Malaysia Medical Centre, Kuala Lumpur, Malaysia e-mail: chingsoongkhoo@gmail.com

Received: May 25, 2020 Accepted: July 26, 2020 •DOI: 10.4274/balkanmedj.galenos.2020.2020.5.208

Available at www.balkanmedicaljournal.org

ORCID iDs of the authors: C.Y.S. 0000-0002-9797-6207; C.S.K. 0000-0002-6756-0411; R.M. 0000-0001-8423-707X; J.N.C. 0000-0001-8229-5276.

Cite this article as:

Sim CY, Khoo CS, Mustafar R, Chai JN. A Double Whammy of Mycotic Aneurysms and Acquired Dysfibrinogenemia in a Patient with Septicemia. Balkan Med J 2021;38:55-6

Copyright@Author(s) - Available online at http://balkanmedicaljournal.org/ 


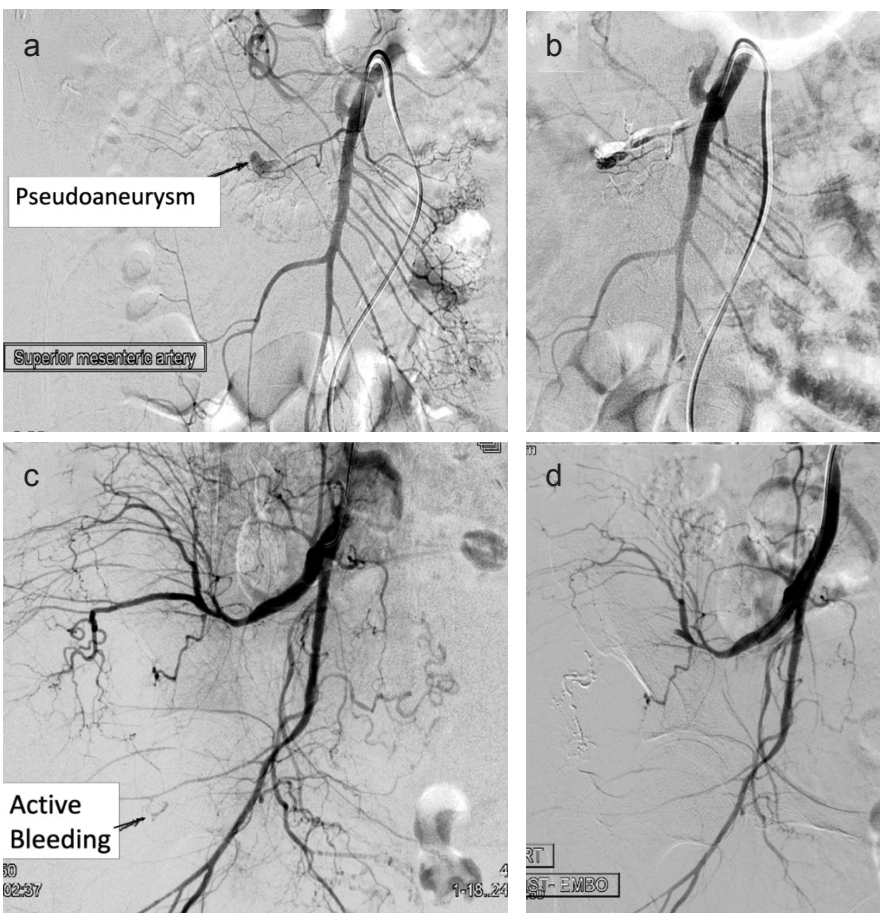

FIG. 2. a-d. (A) Digital subtraction angiogram (DSA) of the superior mesentery artery shows a pseudoaneurysm arising from the right inferior pancreaticoduodenal artery (black arrow). (B) Post-embolization superior mesentery angiogram shows no contrast flow into the pseudoaneurysm. (C) DSA of the right common iliac artery shows active contrast blush (black arrow) seen from a branch of the right inferior gluteal artery. (D) Post-embolization angiogram shows no active bleeding

(Figure 2. c, d). She was discharged on the $26^{\text {th }}$ day of admission without any major complications. Written informed consent was obtained from the patient.

Staphylococci and streptococci are the most common causative pathogens for mycotic aneurysms (1). Aneurysms caused by group A streptococcal septicemia usually affect the large vessels, such as the aorta (2). Our patient developed 2 aneurysms along the right inferior pancreaticoduodenal and right inferior gluteal arteries, which were relatively uncommon. The management of an infected aneurysm is individualized and largely depends on the characteristics of the aneurysm and the patient. Treatment options include open surgery, endovascular stent placement, endovascular embolization, medical therapy, or a combination of any of these $(3,4)$. Any abnormality of fibrinogen can cause hemorrhage, thrombosis, or both. Dysfibrinogenemia is a condition associated with prolonged TT or low fibrinogen level. If TT is prolonged, fibrinogen activity-to-antigen ratio test is performed to diagnose dysfibrinogenemia (5). Acquired dysfibrinogenemia occurs most often in patients with severe liver disorder, producing abnormal fibrinogen molecules. Dysfibrinogenemia may also be associated with cancer, most commonly being liver tumors. Auto-antibodies inhibiting specific functions of fibrinogen have been described in systemic lupus erythematosus, ulcerative colitis, and multiple myeloma (6). Our patient likely had auto-antibodies interfering with the fibrinogen activity owing to her recent relapsed immunoglobulin A nephropathy. This was further supported by her prolonged aPTT, which was not corrected with the mixing test. Concurrent occurrence of these 2 medical conditions in a patient is extremely rare and, to the best of our knowledge, has never been reported. This case highlights the importance of suspecting aneurysm-related bleeding in uncommon locations to avoid treatment delay in potentially reversible and life-saving conditions.

Patient Consent for Publication: Written informed consent was obtained from the patient.

Data-sharing statement: N/A.

Author Contributions: Concept - C.Y.S., C.S.K., R.M., J.N.C.; Design - C.Y.S., C.S.K., R.M., J.N.C.; Data Collection and/or Processing - C.Y.S., C.S.K., R.M., J.N.C.; Analysis and/or Interpretation - C.Y.S., C.S.K., R.M., J.N.C.; Literature Search - C.Y.S., C.S.K., R.M., J.N.C.; Writing Manuscript - C.Y.S., C.S.K., R.M., J.N.C.; Critical Review - C.Y.S., C.S.K., R.M., J.N.C.

Conflict of Interest: The authors have no conflicts of interest to declare.

Funding: The authors declared that this study has received no financial support.

\section{REFERENCES}

1. Lee WK, Mossop PJ, Little AF, Fitt GJ, Vrazas JI, Hoang JK, et al. Infected (mycotic) aneurysms: Spectrum of imaging appearances and management. Radiographics 2008;28:1853-68. [Crossref]

2. Biswas JS, Lyons OT, Bell RE, Price N. Extra-aortic mycotic aneurysm due to group A Streptococcus after pharyngitis. J Clin Microbiol 2013;51:2797-9. [Crossref]

3. Colville J, Madan M, Bashaeb K, Ibrahim R, Sibanda A. Endovascular management of a mycotic group A streptococcal abdominal aortic dissection. BJR Case Rep. 2017;3:20150332. [Crossref]

4. Kaufman JA, Lee MJ. Vascular and Interventional Radiology: The Requisites E-Book: Elsevier Health Sciences; 2013.

5. Cunningham MT, Brandt JT, Laposata M, Olson JD. Laboratory diagnosis of dysfibrinogenemia. Arch Pathol Lab Med 2002;126:499-505.

6. Dear A, Brennan SO, Sheat MJ, Faed JM, George PM. Acquired dysfibrinogenemia caused by monoclonal production of immunoglobulin lambda light chain. Haematologica 2007;92:e111-7. [Crossref] 